\title{
Highly Accurate Current Response Measurements of Insulation Materials for HVDC Applications
}

\section{Conference Paper}

\section{Author(s):}

Tschentscher, Malte; Franck, Christian (i)

Publication date:

2015-10

Permanent link:

https://doi.org/10.3929/ethz-b-000103978

Rights / license:

In Copyright - Non-Commercial Use Permitted

Originally published in:

https://doi.org/10.1109/ICPADM.2015.7295245 


\title{
Highly Accurate Current Response Measurements of Insulation Materials for HVDC Applications
}

\author{
Malte Tschentscher, Christian M. Franck \\ High Voltage Laboratory, ETH Zurich \\ 8092 Zurich, Switzerland
}

\begin{abstract}
This paper presents a current measurement setup to determine polarization and conduction processes of solid insulation materials under HVDC stress. The setup enables simultaneous polarization, depolarization, and conduction current measurements of up to four material samples. The volume or surface current measurements are performed in a pressure vessel, adjustable to the whole range of possible longterm operating conditions present in gas-insulated equipment. Besides discussing the electrical and mechanical construction, this contribution addresses restrictions of the measurement range caused by an insufficient signal-to-noise ratio.
\end{abstract}

Keywords- Polarization measurements; Conductivity measurement; Surface resistance; HVDC transmission

\section{INTRODUCTION}

Both development and design of reliable gas-insulated equipment for HVDC differ significantly from that optimized for HVAC. Phenomena such as surface charge accumulation along with temperature-, field-, and humidity-dependent material properties are dominant in determining the electrical field gradient $[1,2,3,4]$. In order to calculate the electrical field stress in gas-insulated HVDC equipment, knowledge of both conduction and polarization in the bulk and at the insulator surface is essential $[5,6]$. Voltage step excitations result in a time-dependent current response at the electrodes. The time-dependent response is caused by charge transport and polarization in the insulation. Both time-dependent polarization and steady-state currents, i.e. residual currents after relaxation of transient phenomena, are an integral part of insulation characterization. Studies on commonly used solid insulations such as filled/unfilled epoxy resins [7], reinforced thermoplastics, or resin impregnated papers/synthetics have shown transition times to steady-state currents in the order of hours up to months. A large number of the materials response with very slow time-varying currents following power-laws $\propto \mathrm{t}^{-\mathrm{n}}$ with small values of $\mathrm{n}(0<\mathrm{n}<1)$ [8].

The information obtained by frequency domain (FD) measurements of these insulation materials is typically restricted to minimum frequencies of $10^{-3} \mathrm{~Hz}$. Therefore, current response measurements to a voltage step excitation in the time-domain (TD) are a powerful technique for the understanding of very slow polarization and conduction processes. Nevertheless, the transient characterization of highly resistive insulation materials under HVDC stress is challenging in terms of measurement range, accuracy, and precision. The implication of the transient current response due to fast polarization processes, followed by very low and slow timevarying currents, requires current measurements in a range of $10^{-3} \mathrm{~A}$ to $10^{-15} \mathrm{~A}$. Some measurement setups described in literature show noise levels above $10^{-12} \mathrm{~A}$ that compromise the lower end of the measurement range $[3,9,10]$. In other studies, built-in low-noise voltage sources of Keithley ${ }^{\circledR}$ electrometer are used to reduce the noise caused by voltage harmonics, limiting the maximum voltages to $1 \mathrm{kV}[4,11]$.

In order to analyze if an insulation material qualifies for gas-insulated HVDC equipment, it is essential to investigate its behavior in all possible operation conditions. In case of gasinsulated equipment, the temperature at the solid insulation may range from -20 to $90{ }^{\circ} \mathrm{C}$ and the $\mathrm{DC}$ electric field could reach up to $10 \mathrm{kV} / \mathrm{mm}$ in case of polarity reversals [1].

This paper introduces a measurement setup for highly accurate current response measurements to voltage step excitations after a sudden DC application, removal, or reversal. The setup enables parallel investigations of up to four insulation material samples within the entire range of possible operating conditions present in gas-insulated equipment.

\section{EXPERIMENTAL SETUP}

\section{A. Electric Circuit}

The current measurement setup is shown in Figure 1. The high voltage DC source (FUG HCN 140-35000), programmable for voltages up to $\pm 35 \mathrm{kV}$, consists of a grainacher cascade.

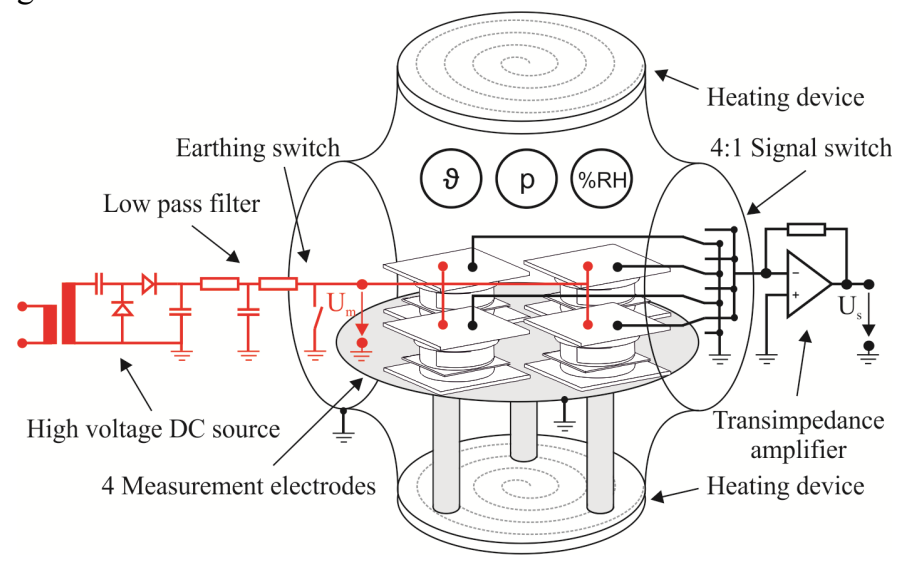

Fig. 1. Measurement setup to determine polarization and conduction processes of solid insulation under HVDC stress 
Residual AC components with amplitudes of $200 \mathrm{mV}_{\mathrm{pp}}$, a minimum frequency of $50 \mathrm{~Hz}$, and harmonics of a multiple of $40 \mathrm{kHz}$ superimpose the high voltage output signal. Due to the setup capacity these disturbances may cause displacement currents reaching up to $10^{-6} \mathrm{~A}$ in the measurement signal. Therefore, the HV signal is filtered by a passive first order low-pass filter consisting of a $10 \mathrm{M} \Omega$ or $50 \mathrm{M} \Omega$ resistor and a $5 \mu \mathrm{F}$ capacitor (rated voltage: $20 \mathrm{kV}_{\mathrm{DC}}$ ). The filter limits the voltage harmonics to a maximum amplitude of $5 \mu \mathrm{V}_{\mathrm{pp}}$ and damps the displacement currents to a negligible level $(<\mathrm{fA})$. An alternative solution is the installation of an active HV lowpass filter. The implementation had been tested but was rejected as it caused further high- frequency interferences.

Figure 2 shows the guard electrode system used for polarization and conduction measurements of solid insulation materials. The aluminium electrodes can be used for both surface and volume-current measurements. For surface current measurements, the outer ring electrode $\left(\mathrm{d}_{\mathrm{re}}=110 \mathrm{~mm}\right.$ or $108 \mathrm{~mm}$ ) is connected to $\mathrm{HV}$ potential and currents, flowing along the insulator surface to the measurement electrode $\left(\mathrm{d}_{\mathrm{me}}=106 \mathrm{~mm}\right)$, are analysed. For investigations of the volume current, the counter electrode $\left(\mathrm{d}_{\mathrm{ce}}=140 \mathrm{~mm}\right)$ is connected to $\mathrm{HV}$ potential, and the outer ring electrode is grounded (GND). The assembly is centered by a hollow cylinder and an insulating cap of PTFE.

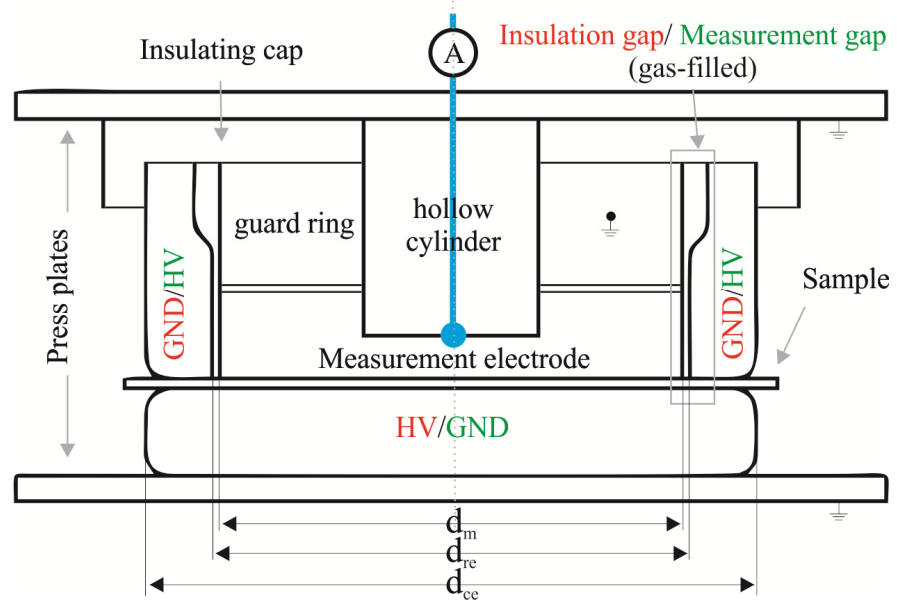

Fig. 2. Contacting method of the cylindrically electrode system for volume current (red) or surface current (green) measurements

To ensure proper contact, $0.5 \mathrm{~mm}$ thick conductive rubber plates are placed between the electrodes and the sample. The rubber compensates surface inhomogeneities and prevents the formation of voids that could cause partial discharges between the sample and the electrodes. The plates are filled with silver plated aluminum particles and add a negligible volume resistivity of $80 \mu \Omega \mathrm{m}$.

A mechanical earthing switch is placed between the electrode assemblies and the HV filter. The switch enables to short-circuit the samples and to measure both polarization and depolarization currents sequentially. The earthing switch is separated from the filter by a resistor of $10 \mathrm{M} \Omega$, limiting the discharge currents of the filter capacitor in case of earthing operation or faults within the system.

Furthermore, a switching board has been constructed, connecting electively one of the four samples with the measurement device while earthing the others via resistors. The earthing resistors are equal to the DC input impedance of the measurement device, i.e. ranging from $1 \Omega$ to $1 \mathrm{M} \Omega$. The performance specification of the switch is determined by its system impact. Firstly, the control circuit of the switch must not disturb the measurement signal. Therefore, either the coil voltage of the relays must be negligible during measurements or the coil-electrode resistance of the switch must be high enough to limit the interference current to orders below the measurement signal. Secondly, no significant leakage current from the signal path to earth must occur. Therefore, the contact to ground resistance of the switching relays must be high compared to the DC input impedance of the measurement device. Thirdly, interferences between the signal paths must not take place during measurements. Hence, the contact to contact resistance of the switching relays must be large compared to the DC input impedance of the measurement device. Lastly, the connection state, "open" or "closed", must be uniquely defined for each switching relay at any time. A toggle relay, for instance, would not satisfy this requirement and could lead to overvoltages within the switch. In accordance with these requirements bi-stable reed relays produced by Meder (BE05-1E66-M) are used, providing a switching coil-contact resistance of $1 \mathrm{~T} \Omega$, minimum coil to ground isolation voltage of $2 \mathrm{kV}$, and a maximum switching current of $0.5 \mathrm{~A}$. The four inputs to one output switching board consists of eight relays and their respective drives. It is controlled by means of four TTL signals. The signals are decoded on the board and define the "open" or "closed" operation of each relay, giving access to 16 switching states. Any disturbance from the power supply is prevented by running the board with an insulated supply and by exclusively applying the supply voltage during switching operations. Resistors ensure the discharging of every board capacity and de-energize the relay drives during current measurements.

The current signals collected at the measurement electrodes are low-pass filtered, converted, and amplified with a transimpedance amplifier (Femto DDPCA-300). The amplifier provides a $\pm 1 \%$ accurate switchable gain from $10^{4}$ to $10^{13} \mathrm{~V} / \mathrm{A}$ and an active low-pass filtering with a variable bandwidth $(-3 \mathrm{~dB})$ of $400 \mathrm{~Hz}$ down to $0.1 \mathrm{~Hz}$. It should be noted that the usability of high gains mainly depends on the maximum input current of the transimpedance amplifier, in full scale including noise. The maximum input current is dependent on the gain and equals $1 \mathrm{pA}$ for a gain of $10^{13} \mathrm{~V} / \mathrm{A}$. Therefore, a strong limitation of the displacement currents is required and achieved by the previously introduced HV lowpass filter between the electrodes and the HV source. The ADconversion of the voltage output signal of the transimpedance amplifier is done with a digital multimeter (Fluke 8845A). Digital filtering of the multimeter output further reduces noise by averaging readings.

\section{B. Mechanical Construction and Temperature Control}

Four electrode assemblies are placed in a pressurecontrolled vessel with nominal working pressures up to $0.58 \mathrm{MPa}$. A pressure sensor (Wika D-10-P) and a humidity and temperature transmitter (Vaisala HMT318) continuously $\log$ the pressure $(\mathrm{p})$, temperature $(\vartheta)$, and relative humidity $(\% \mathrm{RH})$ during current measurements. The pressure sensor is 
sized for a working pressure up to $1.6 \mathrm{MPa}$, guaranteeing a total measurement error $<0.1 \%$. Over the used range the percentage error of the humidity transmitter is $<2 \% \mathrm{RH}$, and the Pt100 temperature sensor shows an accuracy of $\pm 0.35 \mathrm{~K}$.

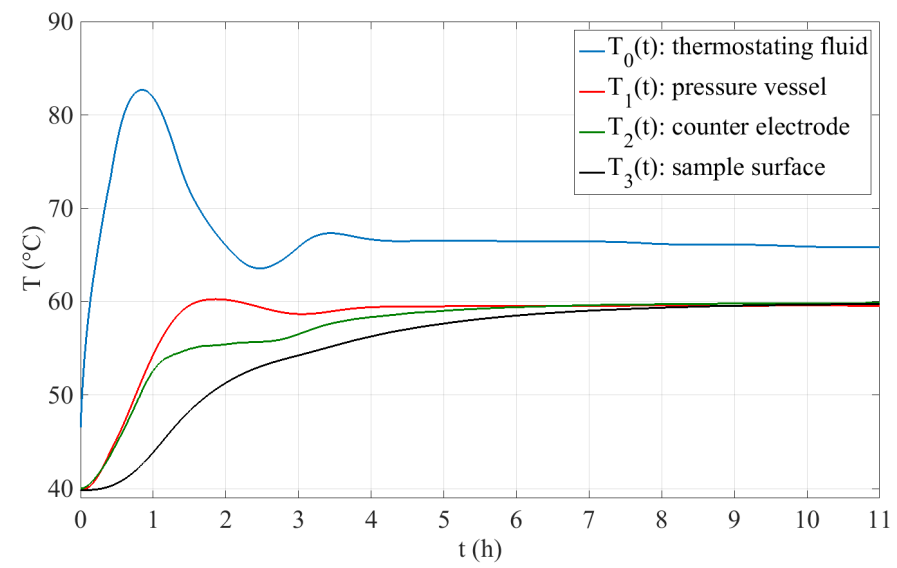

Fig. 3. System temperatures for a control step from $40^{\circ} \mathrm{C}$ to $60^{\circ} \mathrm{C}$

The pressure vessel is temperature-controlled by a liquid filled temperature circuit. A chiller (Huber CC150W), placed in distance to the measurement setup, brings the vessel to a temperature between -20 and $90^{\circ} \mathrm{C}$. This approach avoids mechanical or electrical disturbances of the current measurements. Double layer caps with milled spiral notch between the layers are used to provide a good thermal contact between the liquid circuit and the current measurement setup. One of the caps is placed at the top and one at the bottom of the pressure vessel (see Fig. 1). Figure 3 shows the timedependent temperature measured at different setup parts. The sample surface reaches stable conditions following an exponential change with a heating time constant $\left(\tau_{\text {heat }}\right)$ of 7200 s or a cooling time constant $\left(\tau_{\text {cool }}\right)$ of 14000 s.

\section{SOURCES OF INTERFERENCE}

Noise and temporary disturbances in the low frequency current signal can be due to electrical, mechanical, and thermal interferences. Oscillating disturbances, such as voltage harmonics or permanent mechanical vibrations, can be handled by low-pass filtering of the $\mathrm{HV}$ and measurement signal. Furthermore, any cable connection should be made of low noise cables that shield the signal path. In this setup, cables with carbon-impregnated conductive PTFE layers surrounding the PTFE dielectric are used. The layer dissipates static charges and reduces mechanically generated electrical noise when the cables are bent. Rather challenging is the handling of disturbances caused by thermal interferences. DC offset currents can be introduced by thermoelectric effects. Temperature differences across junctions of dissimilar materials or oxidized contacts in the measurement line cause disturbances in the millivolt range, resulting in pA DC-offset currents. The DC offset currents may lead to the conclusion that the current saturation is due to the end of transient relaxation phenomena. Therefore, the measured currents should be at least one order of magnitude higher than the oscillating disturbances. A good electrical insulation to ground, of the low voltage setup components and connections between the electrodes and the measurement device is essential. It decreases the offset currents as the circuit for thermally induced currents is closed by the low voltage insulation resistances and the DC input impedance of the measurement device. Furthermore, junctions should be avoided or their number at least minimized. Especially, joining of oxidized copper conductors can produce strong DC disturbances as the Seebeck coefficient for such junctions $(\mathrm{Cu}-$ $\mathrm{CuO}$ ) is approximately $1 \mathrm{mV} /{ }^{\circ} \mathrm{C}$, three orders of magnitude higher than for $\mathrm{Cu}-\mathrm{Cu}$. Nevertheless, a voltage drift of the source can cause low frequent displacement currents. Therefore, the voltage drift (dU/dt) times the sample capacity must be small compared to the measurement signal.

\section{MEASURement Performance}

In order to assess the setup performance, three representative current measurements are shown in figure 4 . The vessel was pressurized with $0.45 \mathrm{MPa} \mathrm{SF}_{6}$ to avoid an electrical breakdown of the gaseous insulation.

Two electrode units were connected for surface current measurements, as shown in figure 2. One electrode was filled with a material sample (MS) of epoxy resin and one was left empty to investigate if charge injection (CI) occurs at metal-gas interfaces of the assembly. Furthermore, one measurement channel was high resistive capped (HRC) directly at the input of the switch, to measure how much interference coupling of the three channels occurs on the switching board. Figure 4 shows the raw measurement data and a filtered curve that has been collected by filtering the observed data $\left(20^{\circ} \mathrm{C}\right.$ and $2 \mathrm{kV} / \mathrm{mm}$ ) with a 200 data points moving average (MA) filter. The measurement was performed sequentially on each electrode, with a sample rate of $2 \mathrm{~Hz}$ and collection time of 60 data points.

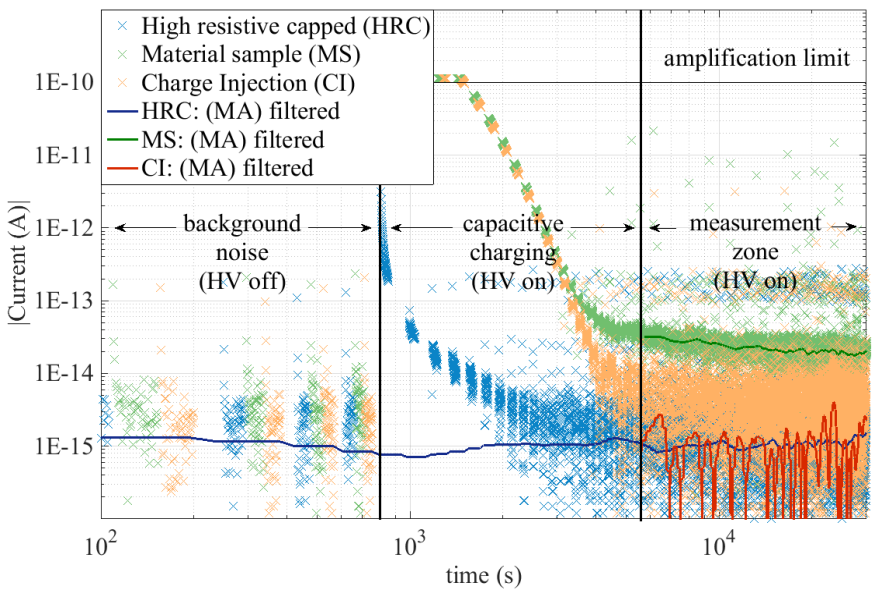

Fig. 4. Surface current measurements of three representative contact topologies at $20^{\circ} \mathrm{C}, 2 \mathrm{kV} / \mathrm{mm}$, and a measurement gain of $10^{11} \mathrm{~V} / \mathrm{A}$

The observed measurement behavior can be divided into three time windows. Firstly, the background noise validation takes place when no $\mathrm{HV}$ signal is applied to the sample. Furthermore, this time window shows whether thermal interferences take place and have to be considered or not. Secondly, the voltage rise time of the setup, starting from the moment that $\mathrm{HV}$ is applied until the measurement voltage $\left(\mathrm{U}_{\mathrm{m}}\right)$ reaches stable conditions, strongly depends on the charging 
time constant of the HV filter. Lastly, the measurement zone enables conclusive identification of sample characteristics. Prior to every measurement, a background noise validation is performed showing a typically filtered noise level of $\pm 1 \mathrm{fA}$. In the moment that $\mathrm{HV}$ is applied, the observed currents jump by orders of magnitude. Capacitive charging of the setup takes place and can even be noticed in the capped channel due to capacitive couplings on the switching board. In the discussed case, we fixed the gain of the transimpedance amplifier to $10^{11} \mathrm{~V} / \mathrm{A}$. This led to an exceeding of the amplification limit during charging, and a saturation of the current amplifier could be seen. The voltage rise time of $U_{m}$ applied to the electrodes may be significantly reduced by pre-charging the HV filter and closing a switch between the HV filter and electrodes afterwards. The switch enables to investigate relaxation times down to $10 \mathrm{~s}$. Furthermore, it is possible to adjust the gain during operation to avoid saturation of the transimpedance amplifier.

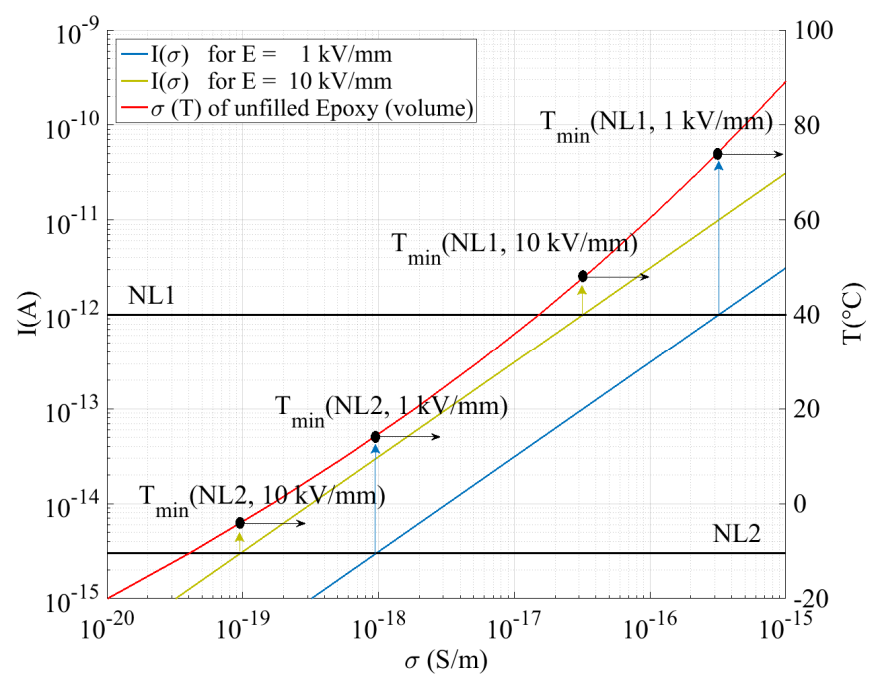

Fig. 5. Evaluation of the accessible measurement range (NL1=1 pA and $\mathrm{NL2}=3 \mathrm{fA}$ ) for a unfilled epoxy resin [11]

The accessible temperature range of solid material investigations is strongly influenced by noise, disturbing the measurement signal. An insufficient signal-to-noise ratio will compromise the lower end of the measurement range and in turn restrict the significance of the investigation. Figure 5 shows the calculated conduction currents of a solid material plate sample, flowing between the outer ring electrode and the measurement electrode, as a function of the volume conductivity $(\sigma)$. The field dependence of the conduction current is neglected and surface conductivity is set equal to the volume conductivity. By following the grid lines, from intersection of the noise levels and the calculated measurement currents to the exponential Arrhenius fit of an example unfilled epoxy resin [11], the minimum accessible temperature for current observations can be found. In this example, the minimum temperature, allowing current observations for a noise level of $1 \mathrm{pA}$ (NL1) and a field stress of $1 \mathrm{kV} / \mathrm{mm}$, is approximately $75^{\circ} \mathrm{C}$. The same material could be investigated for an improved noise level of $3 \mathrm{fA}$ (NL2) down to a temperature of $18{ }^{\circ} \mathrm{C}$. For a field stress of
$10 \mathrm{kV} / \mathrm{mm}$, this setup enables investigations of the conduction, polarization, and injection currents down to a temperature of approximately $-3{ }^{\circ} \mathrm{C}$.

\section{CONCLUSION}

A measurement setup has been designed for highly accurate and precise current measurements under controlled conditions. By means of interference suppression, the configuration enables current measurements down to amplitudes of $\pm 3 \mathrm{fA}$. Measurements can be performed at all possible long-term operating conditions present in gas-insulated equipment. The temperature range extends from -20 to $90^{\circ} \mathrm{C}$. Furthermore, voltages up to $\pm 20 \mathrm{kV}$ can be investigated. The setup gives access to up to four simultaneous current measurements, using only one measurement device. Particularly for measurements of slow time-varying currents, time can be a limiting factor. The signal switch enables to increase the number of investigations by a factor of 4 and consequently reduces the time for analyses equally. Furthermore, simultaneous measurements of volume, surface, and charge injection currents are possible. This approach enables to exclude misinterpretations of current variations caused by environmental influences.

\section{ACKNOWLEDGMENT}

The authors gratefully acknowledge the technical help and financial support of ABB Switzerland Ltd.

\section{REFERENCES}

[1] H. Fujinami, T. Takuma, M. Yashima and T. Kawamoto, "Mechanism and effect of dc charge accumulation on $\mathrm{SF}_{6}$ gas insulated spacers", IEEE PES Winter Meeting, 89 WM 076-1 PWRD, 1989.

[2] B. Weedy, "DC Conductivity of Voltalit Epoxy Spacers in $\mathrm{SF}_{6}$, “, IEE hc., Vol. 132, pt. A, pp. 450-454, 1985.

[3] H. Yahyaoui, P. Notingher, S. Agnel, Y. Kieffel, A. Girodet, "Analysis of Conduction Mechanisms in Alumina-Filled Epoxy Resin under dc Field and Temperature", IEEE Conf. Electr. Insul. Dielectr. Phenomena (CEIDP), pp. 667-670, 2013.

[4] L. Zavattoni, O. Lesaint, O. Gallot-Lavallée, "Surface resistance measurements on epoxy composites: Influence of surrounding gas, pressure, temperature, and analysis of the origin of measured currents", IEEE Conf. on Solid Dielectrics (ICSD), pp. 370-373, 2013.

[5] B. Lutz, „Einflussfaktoren auf die elektrische Feldverteilung in Isoliersystemen mit polymeren Isolierstoffen bei Gleichspannungsbelastung“, Ph.D. thesis, Technical University Munich TUM, 2011.

[6] R. Lovell, "Decaying and steady currents in an epoxy polymer at high electric fields“, J. Phys. D.: Appl. Phys., Vol. 7, 1974.

[7] C. Guillermin, P. Rain and S. Rowe, "Transient and steady state currents in epoxy resin", J. Appl. Phys. D: Vol.39, pp. 515-524, 2006.

[8] A.K. Jonscher, "Dielectric relaxation in solids", Chelsea Dielectrics Press, London, 1983.

[9] B. Lutz, J. Kindersberger, "Influence of absorbed water on volume resistivity of epoxy resin insulators", Conference on solid dielectrics, Germany, 2010.

[10] C. Zou, J.C. Fothergill, S. Zhang, X. Zhou "DC conduction mechanisms in epoxy nanocomposites under the humid environment", International Conference on Solid Dielectrics, Potsdam, Germany, 2010.

[11] B. Lutz and J. Kindersberger, "Surface Charge Accumulation on Cylindrical Polymeric Model Insulators in Air: Simulation and Measurement", IEEE Trans. Dielectr. Electr. Insul, Vol. 18, pp. 20402048, 2011. 